\title{
Cubic B-spline Least-square Method Combine with a Quadratic Weight Function for Solving Integro-Differential Equations
}

\author{
Hameeda O. Al-Humedi ${ }^{1}$ and Zahraa Adnan Jameel ${ }^{2}$ \\ ${ }^{1}$ Department of Mathematics, College of Education for Pure Sciences, Basrah University, Basrah, Iraq \\ e-mail: ahameeda722@yahoo.com \\ ${ }^{2}$ Department of Mathematics, College of Education for Pure Sciences, Basrah University, Basrah, Iraq \\ e-mail: zahraad788@yahoo.com
}

\begin{abstract}
In this article, a numerical scheme was implemented for solving the integro-differential equations (IDEs) with the weakly singular kernel by using a new scheme depend on the cubic B-spline least-square method and a quadratic B-spline as a weight function. The numerical results are in suitable agreement with the exact solutions via calculating $L_{2}$ and $L_{\infty}$ norms errors. Theoretically, we discussed the stability evaluation of the current method using the Von-Neumann method, which explained that this technique is unconditionally stable.
\end{abstract}

\section{Introduction}

The integro-differential equations appear in a wide range of disciplines including physics, chemistry and engineering.

Consider the following IDE with a weakly singular kernel:

$u_{t}(x, t)+m u_{x}(x, t)-b u_{x x}(x, t)=\int_{0}^{t} K(t-s) u(x, s) d s+f(x, t), \quad x \in[a, b], t>0$

where $K(t-s)=(t-s)^{-\alpha}, 0<\alpha<1$

Received: January 23, 2020; Accepted: March 10, 2020

2010 Mathematics Subject Classification: 65R20.

Keywords and phrases: B-spline method, least-square method, integro-differential equation, Von-Neumann.

Copyright (C) 2020 Hameeda O. Al-Humedi and Zahraa Adnan Jameel. This is an open access article distributed under the Creative Commons Attribution License, which permits unrestricted use, distribution, and reproduction in any medium, provided the original work is properly cited. 
subject to the initial condition:

$$
u(x, 0)=g_{0}(x), \quad a \leq x \leq b
$$

and the boundary conditions:

$$
u(a, t)=f_{0}(t), \quad u(b, t)=f_{1}(t), \quad t \geq 0
$$

where $g_{0}(x), f_{0}(t), f_{1}(t)$ are known functions and $f(x, t)$ is a smooth function.

The equations (1)-(3) are of fundamental importance in many physical systems, especially those involving fluid flow $[4,16]$.

Dağ and Naci Özer [3] solved the regularized long-wave equation (RLW) numerically by giving a new algorithm based on a kind of space-time least-square finite element method. Kutluay et al. [9] used a least-squares quadratic B-spline finite element method for calculating the one-dimensional Burgers-like equations with appropriate boundary and initial conditions. El Jid [8] utilized the least-squares and Gauss Legendre to find a solution to the integral equations of the second kind. Dağ [2] applied the leastsquares quadratic B-spline finite element method for solved regularized long wave equation (RLW). Dhawan et al. [6] used Galerkin-least square B-spline to solve the advection-diffusion equation. Dhawan et al. [5] presented a numerical method for solving Burgers equation using B-spline finite element method. Shehab et al. [14] utilized least squares method for solving integral equations with multiple time lags. Gardner et al. [7] used the regularized long-wave equation (RLW) equation to solve by a least-squares technique. Nguyen and Reynen [11] presented the space-time least-square finite element scheme for the advection-diffusion problems at moderate to high Peclet numbers. Chakrabarti and Martha [1] resolved of Fredholm integral equations of the second kind by the least-squares method. Wang et al. [18] used approximation least squares method for solved of Volterra-Fredholm integral equations.

In this research, we will present a new scheme depend on cubic B-spline and quadratic B-spline as a weight function to solve IDE (1); also, we will discuss the stability analysis for the present scheme.

\section{Cubic B-spline Least Square with a Quadratic Weight Function}

The least-square formulation in time and space is explained as

$$
\delta \int_{0}^{t} \int_{a}^{b}\left[u_{t}(x, t)+m u_{x}(x, t)-b u_{x x}(x, t)-\int_{0}^{t}(t-s)^{-\propto} u(x, s) d s-f(x, t)\right]^{2} d x d t=0
$$


and the cubic B-spline $C_{m}(x),(m=-1(1) N+1)$, at the knots $x_{m}$ which form a basis over the solution domain $[a, b]$, is defined as [13]

$$
C_{m}(x)=\frac{1}{h^{3}} \begin{cases}\left(x-x_{m-2}\right)^{3}, & \text { if } x \in\left[x_{m-2}, x_{m-1}\right] \\ h^{3}+3 h^{2}\left(x-x_{m-1}\right)+3 h\left(x-x_{m-1}\right)^{2}-3\left(x-x_{m-1}\right)^{3}, & \text { if } x \in\left[x_{m-1}, x_{m}\right] \\ h^{3}+3 h^{2}\left(x_{m+1}-x\right)+3 h\left(x_{m+1}-x\right)^{2}-3\left(x_{m+1}-x\right)^{3}, & \text { if } x \in\left[x_{m}, x_{m+1}\right] \\ \left(x_{m+2}-x\right)^{3}, & \text { if } x \in\left[x_{m+1}, x_{m+2}\right] \\ 0, & \text { otherwise. }\end{cases}
$$

The set of splines $\left(C_{-1}(x), C_{0}(x), \ldots, C_{N}(x), C_{N+1}(x)\right)$ forms a basis for functions defined over $[a, b]$. Consider the approximate solution $U_{N}(x, t)$ to the exact solution $U(x, t)$ given by

$$
U_{N}(x, t)=\sum_{i=-1}^{N+1} C_{i}(x) \sigma_{i}(t),
$$

where $\sigma_{i}$ are unknown time-dependent parameters to be determined from the boundary and weighted residual conditions. We will use the following local coordinate transformation

$$
h \eta=x-x_{m}, \quad 0 \leq \eta \leq 1,
$$

a cubic B-spline shape functions in terms of $\eta$ over the element $\left[x_{m}, x_{m+1}\right]$ that can be defined as

$$
\left\{\begin{array}{l}
C_{m-1}=(1-\eta)^{3} \\
C_{m}=1+3(1-\eta)+3(1-\eta)^{2}-3(1-\eta)^{3} \\
C_{m+1}=1+3 \eta+3 \eta^{2}-3 \eta^{3} \\
C_{m+2}=\eta^{3}
\end{array}\right.
$$

all splines apart from $C_{m-1}, C_{m}, C_{m+1}$ and $C_{m+2}$ are zero over the element $\left[x_{m}, x_{m+1}\right]$ on each time interval $\left[t_{n}, t_{n+1}\right], \Delta t=t^{n+1}-t^{n}$ is a local coordinate $\zeta$, where

$$
t=t^{n}+\zeta \Delta t, \quad 0 \leq \zeta \leq 1
$$

By using the transformations (7) and (9) in equation (4) we obtain

$$
\delta \int_{0}^{1} \int_{0}^{1}\left[u_{\zeta}(x, t)+\frac{m}{h} u_{\eta}(x, t)-\frac{b}{h^{2}} u_{\eta \eta}(x, t)-\int_{0}^{\zeta}(\zeta-s)^{-\propto} u(\eta, s) d s-f(\eta, \zeta)\right]^{2} d \eta d \zeta=0 .
$$

The integral equation takes its minimum value with the variation in $u$ over each element $\left[x_{m}, x_{m+1}\right]$, then 


$$
\begin{aligned}
& \int_{0}^{1} \int_{0}^{1}\left\{[ u _ { \zeta } + \frac { m } { h } u _ { \eta } - \frac { b } { h ^ { 2 } } u _ { \eta \eta } - \int _ { 0 } ^ { \zeta } ( \zeta - s ) ^ { - \propto } u ( \eta , s ) d s - f ( \eta , \zeta ) ] \delta \left[u_{\zeta}+\frac{m}{h} u_{\eta}-\right.\right. \\
& \left.\left.\frac{b}{h^{2}} u_{\eta \eta}-\int_{0}^{\zeta}(\zeta-s)^{-\propto} u(\eta, s) d s-f(\eta, \zeta)\right]\right\} d \eta d \zeta=0 .
\end{aligned}
$$

The least square method turns into a Petrov-Galerkin method with then weight function,

$$
\delta\left[u_{\zeta}+\frac{m}{h} u_{\eta}-\frac{b}{h^{2}} u_{\eta \eta}-\int_{0}^{\zeta}(\zeta-s)^{-\propto} u(\eta, s) d s-f(\eta, \zeta)\right],
$$

the variation of $u$ over the element $\left[x_{m}, x_{m+1}\right]$ defined by

$$
U_{N}(\eta, \zeta)=\sum_{i=m-1}^{m+2} C_{i}(\eta)\left(\sigma_{i}^{n}+\zeta \Delta \sigma_{i}^{n}\right),
$$

where $\sigma_{m-1}(t), \sigma_{m}(t), \sigma_{m+1}(t)$ and $\sigma_{m+2}(t)$ act as element parameters and B-splines $C_{m-1}(\eta), C_{m}(\eta), C_{m+1}(\eta)$ and $C_{m+2}(\eta)$ as element shape functions. The spline $C_{m}(x)$ vanishes outside the interval $\left[x_{m-2}, x_{m+2}\right]$. So, the value of $U$ with its first and second derivatives $U^{\prime}, U^{\prime \prime}$ respectively at the knots, $x_{m}$ which is determined in terms of element parameters $\sigma_{m}$ by

$$
\left.\begin{array}{l}
U_{m}=U\left(x_{m}\right)=\sigma_{m-1}+4 \sigma_{m}+\sigma_{m+1} \\
U_{m}^{\prime}=U^{\prime}\left(x_{m}\right)=\frac{3}{h}\left(\sigma_{m-1}-\sigma_{m+1}\right) \\
U_{m}^{\prime \prime}=U^{\prime \prime}\left(x_{m}\right)=\frac{6}{h^{2}}\left(\sigma_{m-1}-2 \sigma_{m}+\sigma_{m+1}\right)
\end{array}\right\}
$$

take the weight function, $W$ quadratic B-spline that is defined as

$$
B_{m}(x)=\frac{1}{h^{2}}\left\{\begin{array}{cl}
\left(x_{m+2}-x\right)^{2}-3\left(x_{m+1}-x\right)^{2}+3\left(x_{m}-x\right)^{2}, & \text { if } x \in\left[x_{m-1}, x_{m}\right], \\
\left(x_{m+2}-x\right)^{2}-3\left(x_{m+1}-x\right)^{2}, & \text { if } x \in\left[x_{m}, x_{m+1}\right], \\
\left(x_{m+2}-x\right)^{2}, & \text { if } x \in\left[x_{m+1}, x_{m+2}\right], \\
0, & \text { otherwise. }
\end{array}\right.
$$

Write the weight function as

$$
\delta W=\sum_{i_{2}=m-1}^{m+1} W \Delta \gamma_{i_{2}}=\delta\left(u_{\zeta}+\lambda u_{\eta}-\beta u_{\eta \eta}-\int_{0}^{t}(\zeta-s)^{-\propto} u(\eta, s) d s-f(\eta, \zeta)\right) .
$$

Using the expansion (12) so that

$$
\delta U_{N}(\eta, \zeta)=\sum_{i=m-1}^{m+1} \zeta B_{i}(\eta) \Delta \gamma_{i}^{n},
$$

we get

$$
W=\delta\left(u_{\zeta}+\lambda u_{\eta}-\beta u_{\eta \eta}-\int_{0}^{\zeta}(\zeta-s)^{-\propto} u(\eta, s) d s-f(\eta, \zeta)\right)=B_{i}(\eta)+
$$




$$
\lambda \zeta B_{i}^{\prime}(\eta)-\beta \zeta B_{i_{2}}^{\prime \prime}(\eta)-\int_{0}^{\zeta} s(\zeta-s)^{-\propto} B_{i}(\eta) d s-f(\eta, \zeta) .
$$

Substitution (16) in equation (11) we get

$$
\begin{aligned}
& \int_{0}^{1} \int_{0}^{1}\left\{[ u _ { \zeta } + \lambda u _ { \eta } - \beta u _ { \eta \eta } - \int _ { 0 } ^ { \zeta } ( \zeta - s ) ^ { - \propto } u ( \eta , s ) d s - f ( \eta , \zeta ) ] \left[B_{i}(\eta)+\right.\right. \\
& \left.\left.\lambda \zeta B_{i}^{\prime}(\eta)-\beta \zeta B_{i}^{\prime \prime}(\eta)-\int_{0}^{\zeta} s(\zeta-s)^{-\propto} B_{i}(\eta) d s-f(\eta, \zeta)\right]\right\} d \eta d \zeta=0 .
\end{aligned}
$$

Substituting (11) in equation (17), integration with respect to $\zeta$ and integration by parts as required leads to the following matrix system of equations for each individual element

$$
\begin{aligned}
& \sum_{j=m-1}^{m+2}\left\{\int _ { 0 } ^ { 1 } \left[\left(B_{i} C_{j}+\left(\beta+\frac{\lambda^{2}}{3}\right) B_{i}^{\prime} C_{j}^{\prime}+\frac{\lambda}{2} B_{i}^{\prime} C_{j}+\frac{\lambda}{2} B_{i} C_{j}^{\prime}-\frac{\lambda \beta}{3} B_{i}^{\prime \prime} C_{j}^{\prime}-\frac{\beta \lambda}{3} B_{i}^{\prime} C_{j}^{\prime \prime}+\right.\right.\right. \\
& \left.\frac{\beta^{2}}{3} B_{i}^{\prime \prime} C_{j}^{\prime \prime}\right)+\left(-2 B_{i} C_{j}-\beta B_{i}^{\prime} C_{j}^{\prime}-\frac{\lambda}{2} B_{i} C_{j}^{\prime}-\frac{\lambda}{2} B_{i}^{\prime} C_{j}\right) \int_{0}^{\zeta} s(\zeta-s)^{-\propto} d s+ \\
& \left(B_{i} C_{j}\right) \int_{0}^{\zeta} \int_{0}^{\zeta} s^{2}(\zeta-s)^{-2 \propto} d s d s+\int_{0}^{1}\left[\left(-C_{j}-\lambda \zeta C_{j}^{\prime}+\beta \zeta C_{j}^{\prime \prime}+C_{j} \int_{0}^{\zeta} s(\zeta-\right.\right. \\
& \left.\left.\left.s)^{-\propto} d s\right) f(\eta, \zeta)\right] d \zeta\right] d \eta+ \\
& \left.\left[\left(-\left.\frac{\beta}{2} B_{i}^{\prime} C_{j}\right|_{0} ^{1}-\left.\frac{\beta}{2} B_{i} C_{j}^{\prime}\right|_{0} ^{1}\right)+\left(\left.\frac{\beta}{2} B_{i} C_{j}^{\prime}\right|_{0} ^{1}+\left.\frac{\beta}{2} B_{i}^{\prime} C_{j}\right|_{0} ^{1}\right) \int_{0}^{\zeta} s(\zeta-s)^{-\propto} d s\right]\right\} \Delta \sigma_{j} \\
& +\sum_{j=m-1}^{m+2}\left\{\int _ { 0 } ^ { 1 } \left[\left(\beta+\frac{\lambda^{2}}{2}\right) B_{i}^{\prime} C_{j}^{\prime}+\lambda B_{i} C_{j}^{\prime}-\frac{\beta \lambda}{2} B_{i}^{\prime} C_{j}^{\prime \prime}-\frac{\lambda \beta}{2} B_{i}^{\prime \prime} C_{j}^{\prime}+\frac{\beta^{2}}{2} B_{i}^{\prime \prime} C_{j}^{\prime \prime}+\right.\right. \\
& \left(-\lambda B_{i} C_{j}^{\prime}-\beta B_{i}^{\prime} C_{j}^{\prime}\right) \int_{0}^{\zeta} s(\zeta-s)^{-\propto} d s+\left(-B_{i} C_{j}-\frac{\lambda}{2} B_{i}^{\prime} C_{j}-\frac{\beta}{2} B_{i}^{\prime} C_{j}^{\prime}\right) \int_{0}^{\zeta}(\zeta-s)^{-\propto} d s+ \\
& \left(B_{i} C_{j}\right) \int_{0}^{\zeta} \int_{0}^{\zeta} s(\zeta-s)^{-2 \propto} d s d s+ \\
& \left.\int_{0}^{1}\left[\left(-\lambda C_{j}^{\prime}+\beta C_{j}^{\prime \prime}+C_{j} \int_{0}^{\zeta}(\zeta-s)^{-\propto} d s\right) f(\eta, \zeta)\right] d \zeta\right] d \eta+\left[\left(-\left.\beta B_{i} C_{j}^{\prime}\right|_{0} ^{1}\right)+\right. \\
& \left.\left.\left(\left.\beta B_{i} C_{j}^{\prime}\right|_{0} ^{1}\right) \int_{0}^{\zeta} s(\zeta-s)^{-\propto} d s+\left(\left.\frac{\beta}{2} B_{i}^{\prime} C_{j}\right|_{0} ^{1}\right) \int_{0}^{\zeta}(\zeta-s)^{-\propto} d s\right]\right\} \sigma_{j}+\int_{0}^{1} \int_{0}^{1}\left(-B_{i}-\right. \\
& \left.\lambda \zeta B_{i}^{\prime}+\beta \zeta B_{i}^{\prime \prime}+B_{i} \int_{0}^{\zeta} s(\zeta-s)^{-\propto} d s+f(\eta, \zeta)\right) f(\eta, \zeta) d \eta d \zeta=0 .
\end{aligned}
$$

The equation (18) can be written in a matrix form as follows:

$$
\begin{aligned}
& {\left[\left(A_{k}^{e}+\left(\beta+\frac{\lambda^{2}}{3}\right) B_{k}^{e}+\frac{\beta^{2}}{3} C_{k}^{e}+\frac{\lambda}{2} D_{k}^{e}+\frac{\lambda}{2}\left(D_{k}^{e}\right)^{T}-\frac{\beta \lambda}{3} E_{k}^{e}-\frac{\lambda \beta}{3}\left(E_{k}^{e}\right)^{T}-\frac{\beta}{2}\left(F_{k}^{e}\right)^{T}-\right.\right.} \\
& \left.\frac{\beta}{2} F_{k}^{e}\right)+\left(-2 A_{k}^{e}-\beta B_{k}^{e}-\frac{\lambda}{2} D_{k}^{e}-\frac{\lambda}{2}\left(D_{k}^{e}\right)^{T}+\frac{\beta}{2} F_{k}^{e}+\frac{\beta}{2}\left(F_{k}^{e}\right)^{T}\right) \int_{0}^{\zeta} s(\zeta-s)^{-\alpha} d s+ \\
& \left(A_{k}^{e} \int_{0}^{\zeta} \int_{0}^{\zeta} s^{2}(\zeta-s)^{-2 \alpha} d s d s\right)+ \\
& \int_{0}^{1} \int_{0}^{1}\left(-C_{j}-\lambda \zeta C_{j}^{\prime}+\beta \zeta C_{j}^{\prime \prime}+\beta \zeta C_{j}^{\prime \prime}\right) f(\eta, \zeta) d \eta d \zeta+\int_{0}^{1} \int_{0}^{1}\left(-C_{j}-\lambda \zeta C_{j}^{\prime}+\beta \zeta C_{j}^{\prime \prime}+\right. \\
& \left.\left.\int_{0}^{\zeta} s(\zeta-s)^{-\alpha} C_{j} d s\right) f(\eta, \zeta) d \eta d \zeta\right] \Delta \sigma^{e}
\end{aligned}
$$


$+\left[\left(\lambda D_{k}^{e}+\frac{\lambda^{2}}{2} B_{k}^{e}-\frac{\lambda \beta}{2}\left(E_{k}^{e}\right)^{T}-\beta F_{k}^{e}+\beta B_{k}^{e}-\frac{\lambda \beta}{2} E_{k}^{e}+\frac{\beta^{2}}{2} C_{k}^{e}\right)+\left(-\lambda D_{k}^{e}+\right.\right.$ $\left.\beta F_{k}^{e}-\beta B_{k}^{e}\right) \int_{0}^{\zeta} s(\zeta-s)^{-\propto} d s+\left(-A_{k}^{e}-\frac{\lambda}{2}\left(D_{k}^{e}\right)^{T}+\frac{\beta}{2} F_{i_{5} j_{5}}^{T}-\frac{\beta}{2} B_{i j}^{e}\right) \int_{0}^{\zeta}(\zeta-s)^{-\propto} d s+$ $\left(A_{k}^{e} \int_{0}^{\zeta} \int_{0}^{\zeta} s(\zeta-s)^{-2 \alpha} d s d s\right)+\int_{0}^{1} \int_{0}^{1}\left(-\lambda C_{j}^{\prime}+\beta C_{j}^{\prime \prime}+\right.$ $\left.\left.\int_{0}^{\zeta}(\zeta-s)^{-\alpha} C_{j} d s\right) f(\eta, \zeta) d \eta d \zeta\right] \sigma^{e}+\int_{0}^{1} \int_{0}^{1}\left(-B_{i}-\lambda \zeta B_{i}^{\prime}+\beta \zeta B_{i}^{\prime \prime}+\int_{0}^{\zeta} s(\zeta-\right.$ $\left.s)^{-\propto} B_{i} d s\right) f(\eta, \zeta) d \eta d \zeta+\int_{0}^{1} \int_{0}^{1} f(\eta, \zeta) f(\eta, \zeta) d \eta d \zeta=0$,

where $\sigma^{e}=\left(\sigma_{m-1}, \sigma_{m}, \sigma_{m+1}, \sigma_{m+2}\right)$ element parameters and matrices are given by the following

$$
\begin{gathered}
A_{k}^{e}=\int_{0}^{1} B_{i} C_{j} d \eta=\frac{1}{60}\left[\begin{array}{cccc}
10 & 71 & 38 & 1 \\
19 & 221 & 221 & 19 \\
1 & 38 & 71 & 10
\end{array}\right], \\
B_{k}^{e}=\int_{0}^{1} B_{i}^{\prime} C_{j}^{\prime} d \eta=\frac{1}{2}\left[\begin{array}{cccc}
3 & 5 & -7 & -1 \\
-2 & 2 & 2 & -2 \\
-1 & -7 & 5 & 3
\end{array}\right], \\
C_{k}^{e}=\int_{0}^{1} B_{i}^{\prime \prime} C_{j}^{\prime \prime} d \eta=\left[\begin{array}{cccc}
6 & -6 & -6 & 6 \\
-12 & 12 & 12 & -12 \\
6 & -6 & -6 & 6
\end{array}\right], \\
D_{k}^{e}=\int_{0}^{1} B_{i} C_{j}^{\prime} d \eta=\frac{1}{10}\left[\begin{array}{cccc}
-6 & -7 & 12 & 1 \\
-13 & -41 & 41 & 13 \\
-1 & -12 & 7 & 6
\end{array}\right], \\
E_{k}^{e}=\int_{0}^{1} B_{i}^{\prime} C_{j}^{\prime \prime} d \eta=\left[\begin{array}{cccc}
-4 & 6 & 0 & -2 \\
2 & -6 & 6 & -2 \\
2 & 0 & -6 & 4
\end{array}\right], \\
F_{k}^{e}=B_{i} C_{j}^{\prime} l_{0}^{1}=3\left[\begin{array}{cccc}
1 & 0 & -1 & 0 \\
1 & -1 & -1 & 1 \\
0 & -1 & 0 & 1
\end{array}\right],
\end{gathered}
$$

where $i$ and $j$ take the value $m-1, m, m+1, m+2$ from element $\left[x_{m}, x_{m+1}\right]$.

Assembling all contribution from all element yields the global system of equations

$$
\begin{aligned}
& {\left[\left(A_{k}+\frac{\lambda}{2} D_{k}^{T}-\frac{\beta}{2} F_{k}^{T}+\beta B_{k}+\frac{\lambda}{2} D_{k}+\frac{\lambda^{2}}{3} B_{k}-\frac{\lambda \beta}{3} E_{k}^{T}-\frac{\beta}{2} F_{k}-\frac{\beta \lambda}{3} E_{k}+\frac{\beta^{2}}{3} C_{k}\right)+\right.} \\
& \left(-2 A_{k}-\frac{\lambda}{2} D_{k}+\frac{\beta}{2} F_{k}-\beta B_{k}-\frac{\lambda}{2} D_{k}^{T}+\frac{\beta}{2} F_{k}^{T}\right) \int_{0}^{\zeta} s(\zeta-s)^{-\propto} d s+\left(A_{k} \int_{0}^{\zeta} \int_{0}^{\zeta} s^{2}(\zeta-\right. \\
& \left.s)^{-2 \propto} d s d s\right)+\int_{0}^{1} \int_{0}^{1}\left(-C_{j}-\lambda \zeta C_{j_{3}}^{\prime}+\beta \zeta C_{j}^{\prime \prime}+\beta \zeta C_{j}^{\prime \prime}\right) f(\eta, \zeta) d \eta d \zeta+\int_{0}^{1} \int_{0}^{1}\left(-C_{j}-\right. \\
& \left.\left.\lambda \zeta C_{j}^{\prime}+\beta \zeta C_{j}^{\prime \prime}+\int_{0}^{\zeta} s(\zeta-s)^{-\propto} C_{j} d s\right) f(\eta, \zeta) d \eta d \zeta\right] \Delta \sigma
\end{aligned}
$$


$+\left[\left(\lambda D_{k}+\frac{\lambda^{2}}{2} B_{k}-\frac{\lambda \beta}{2} E_{k}^{T}-\beta F_{k}+\beta B_{k}-\frac{\lambda \beta}{2} E_{k}+\frac{\beta^{2}}{2} C_{k}\right)+\left(-\lambda D_{k}+\beta F_{k}-\right.\right.$ $\left.\beta B_{k}\right) \int_{0}^{\zeta} s(\zeta-s)^{-\alpha} d s+\left(-A_{k}-\frac{\lambda}{2} D_{k}^{T}+\frac{\beta}{2} F_{k}^{T}-\frac{\beta}{2} B_{k}\right) \int_{0}^{\zeta}(\zeta-s)^{-\alpha} d s+$ $\left(A_{k} \int_{0}^{\zeta} \int_{0}^{\zeta} s(\zeta-s)^{-2 \alpha} d s d s\right)+\int_{0}^{1} \int_{0}^{1}\left(-\lambda C_{j}^{\prime}+\beta j+\right.$ $\left.\left.\int_{0}^{\zeta}(\zeta-s)^{-\alpha} C_{j} d s\right) f(\eta, \zeta) d \eta d \zeta\right] \sigma+\int_{0}^{1} \int_{0}^{1}\left(-B_{i}-\lambda \zeta B_{i}^{\prime}+\beta \zeta B_{i}^{\prime \prime}+\int_{0}^{\zeta} s(\zeta-\right.$

$\left.s)^{-\alpha} B_{i} d s\right) f(\eta, \zeta) d \eta d \zeta+\int_{0}^{1} \int_{0}^{1} f(\eta, \zeta) f(\eta, \zeta) d \eta d \zeta=0$, where $\sigma=\left(\sigma_{0}, \sigma_{1}, \ldots, \sigma_{N}\right)^{T}$ is a global element.

Recognize $\sigma=\sigma^{n}, \Delta \sigma=\sigma^{n+1}-\sigma^{n}$ in the system of equation (20), we obtain $(N+3) \times(N+3)$.

Septa-diagonal matrix the equation (20) is written as follows:

$\left[\left(A_{k}+\left(\beta+\frac{\lambda^{2}}{3}\right) B_{k}+\frac{\lambda}{2} D_{k}+\frac{\lambda}{2} D_{k}^{T}-\frac{\beta \lambda}{3} E_{k}-\frac{\beta \lambda}{3} E_{k}^{T}-\frac{\beta}{2} F_{k}-\frac{\beta}{2} F_{k}^{T}+\frac{\beta^{2}}{3} C_{k}\right)+\right.$ $\left(-2 A_{k}-\beta B_{k}-\frac{\lambda}{2} D_{k}-\frac{\lambda}{2} D_{k}^{T}+\frac{\beta}{2} F_{k}+\frac{\beta}{2} F_{k}^{T}\right) \int_{0}^{\zeta} s(\zeta-s)^{-\alpha} d s+\left(A_{k}\right) \int_{0}^{\zeta} \int_{0}^{\zeta} s^{2}(\zeta-$ $\left.s)^{-2 \propto} d s d s+\int_{0}^{1} \int_{0}^{1}\left(-C_{j}-\lambda \zeta C_{j}^{\prime}+\beta \zeta C_{j}^{\prime \prime}+C_{j} \int_{0}^{\zeta} s(\zeta-s)^{-\alpha} d s\right) f(\eta, \zeta) d \eta d \zeta\right] \sigma^{n+1}$ $=\left[\left(A_{k}-\frac{\lambda^{2}}{6} B_{k}-\frac{\lambda}{2} D_{k}+\frac{\lambda}{2} D_{k}^{T}+\frac{\beta \lambda}{6} E_{k}+\frac{\beta \lambda}{6} E_{k}^{T}+\frac{\beta}{2} F_{k}-\frac{\beta}{2} F_{k}^{T}-\frac{\beta^{2}}{6} C_{k}\right)-\right.$ $\left(2 A_{k}-\frac{\lambda}{2} D_{k}+\frac{\lambda}{2} D_{k}^{T}+\frac{\beta}{2} F_{k}-\frac{\beta}{2} F_{k}^{T}\right) \int_{0}^{\zeta} s(\zeta-s)^{-\alpha} d s+\left(A_{k}+\frac{\beta}{2} B_{k}+\frac{\lambda}{2} D_{k}^{T}-\right.$ $\left.\frac{\beta}{2} F_{k}^{T}\right) \int_{0}^{\zeta}(\zeta-s)^{-\alpha} d s-\left(A_{k}\right) \int_{0}^{\zeta} \int_{0}^{\zeta} s(\zeta-s)^{-2 \propto} d s d s+\left(A_{k}\right) \int_{0}^{\zeta} \int_{0}^{\zeta} s^{2}(\zeta-$ $s)^{-2 \propto} d s d s+\int_{0}^{1} \int_{0}^{1}\left(C_{j}+\lambda C_{j}^{\prime}+\lambda \zeta C_{j}^{\prime}-\beta C_{j}^{\prime \prime}-\beta \zeta C_{j}^{\prime \prime}-C_{j} \int_{0}^{\zeta}(\zeta-s)^{-\propto} d s-\right.$ $\left.\left.C_{j} \int_{0}^{\zeta} s(\zeta-s)^{-\alpha} d s\right) f(\eta, \zeta) d \eta d \zeta\right] \sigma^{n}+\int_{0}^{1} \int_{0}^{1}\left(B_{i}+\lambda \zeta B_{i}^{\prime}-\beta \zeta B_{i}^{\prime \prime}-\int_{0}^{\zeta} s(\zeta-\right.$ $\left.s)^{-\propto} B_{i} d s-f(\eta, \zeta)\right) f(\eta, \zeta) d \eta d \zeta$.

The integral equation has a value

$\int_{0}^{\zeta}(\zeta-s)^{-\alpha} d s=\frac{\zeta^{1-\alpha}}{1-\alpha^{\prime}} \int_{0}^{\zeta} s(\zeta-s)^{-\alpha} d s=\frac{\zeta^{2-\alpha}}{\alpha^{2}+3 \alpha+2}$

$\int_{0}^{\zeta} \int_{0}^{\zeta} s^{2}(\zeta-s)^{-2 \alpha} d s d s=\frac{\zeta^{4-2 \alpha}}{-4 \alpha^{3}+12 \alpha^{2}-11 \alpha+3^{\prime}} \int_{0}^{\zeta} \int_{0}^{\zeta} s(\zeta-s)^{-2 \alpha} d s d s=\frac{\zeta^{3-2 \alpha}}{4 \alpha^{2}-6 \alpha+2}$.

The equation (21) can be written as follows: 
$\left[\left(\left(1-2 \frac{\zeta^{2-\alpha}}{\alpha^{2}-3 \alpha+2}+\frac{\zeta^{4-2 \alpha}}{-4 \alpha^{3}+12 \alpha^{2}-11 \alpha+3}\right) A_{k}+\left(\frac{\lambda^{2}}{3}+\beta-\beta \frac{\zeta^{2-\alpha}}{\alpha^{2}-3 \alpha+2}\right) B_{k}+\frac{\beta^{2}}{3} C_{k}+\right.\right.$

$\left(\frac{\lambda}{2}-\frac{\lambda}{2} \frac{\zeta^{2-\alpha}}{\alpha^{2}-3 \alpha+2}\right) D_{k}+\left(\frac{\lambda}{2}-\frac{\lambda}{2} \frac{\zeta^{2-\alpha}}{\alpha^{2}-3 \alpha+2}\right) D_{k}^{T}-\frac{\beta \lambda}{3} E_{k}-\frac{\lambda \beta}{3} E_{k}^{T}+\left(-\frac{\beta}{2}+\frac{\beta}{2} \frac{\zeta^{2-\alpha}}{\alpha^{2}-3 \alpha+2}\right) F_{k}+$

$\left.\left(-\frac{\beta}{2}+\frac{\beta}{2} \frac{\zeta^{2-\alpha}}{\alpha^{2}-3 \alpha+2}\right) F_{k}^{T}\right)-$

$\left.\int_{0}^{1} \int_{0}^{1}\left(C_{j}+\lambda \zeta C_{j}^{\prime}-\beta \zeta C_{j}^{\prime \prime}-\frac{\zeta^{2-\alpha}}{\alpha^{2}-3 \alpha+2} C_{j}\right) f(\eta, \zeta) d \eta d \zeta\right] \sigma^{n+1}$

$=\left[\left(\left(1+\frac{\zeta^{1-\alpha}}{1-\alpha}-2 \frac{\zeta^{2-\alpha}}{\alpha^{2}-3 \alpha+2}-\frac{\zeta^{3-2} \propto}{4 \alpha^{2}-6 \propto+2}+\frac{\zeta^{4-2 \propto}}{-4 \alpha^{3}+12 \alpha^{2}-11 \propto+3}\right) A_{k}+\left(-\frac{\lambda^{2}}{6}+\right.\right.\right.$

$\left.\frac{\beta}{2} \frac{\zeta^{1-\alpha}}{1-\alpha}\right) B_{k}-\frac{\beta^{2}}{6} C_{k}+\left(-\frac{\lambda}{2}+\frac{\lambda}{2} \frac{\zeta^{2-\alpha}}{\alpha^{2}-3 \alpha+2}\right) D_{k}+\left(\frac{\lambda}{2}+\frac{\lambda}{2} \frac{\zeta^{1-\alpha}}{1-\alpha}-\frac{\lambda}{2} \frac{\zeta^{2-\alpha}}{\alpha^{2}-3 \alpha+2}\right) D_{k}^{T}+\frac{\lambda \beta}{6} E_{k}+$

$\left.\frac{\lambda \beta}{6} E_{k}^{T}+\left(\frac{\beta}{2}-\frac{\beta}{2} \frac{\zeta^{2-\alpha}}{\alpha^{2}-3 \alpha+2}\right) F_{k}+\left(-\frac{\beta}{2}-\frac{\beta}{2} \frac{\zeta^{1-\alpha}}{1-\alpha}+\frac{\beta}{2} \frac{\zeta^{2-\alpha}}{\alpha^{2}-3 \alpha+2}\right) F_{k}^{T}\right)+\int_{0}^{1} \int_{0}^{1}\left(C_{j}+\lambda C_{j}^{\prime}+\right.$

$\left.\left.\lambda \zeta C_{j}^{\prime}-\beta C_{j}^{\prime \prime}-\beta \zeta C_{j}^{\prime \prime}-\frac{\zeta^{1-\alpha}}{1-\alpha} C_{j}-C_{j} \frac{\zeta^{2-\alpha}}{\alpha^{2}-3 \alpha+2}\right) f(\eta, \zeta) d \eta d \zeta\right] \sigma^{n}+\int_{0}^{1} \int_{0}^{1}\left(B_{i}+\lambda \zeta B_{i}^{\prime}-\right.$

$\left.\beta \zeta B_{i}^{\prime \prime}-B_{i} \frac{\zeta^{2-\alpha}}{\alpha^{2}-3 \alpha+2}-f(\eta, \zeta)\right) f(\eta, \zeta) d \eta d \zeta$.

From the equation (22), we get system of $(N+1)$ linear equation with $(N+$ 3 ) unknowns. We apply the initial condition $u(x, 0)=g_{0}(x)$ to the equation (14) makes the matrix equation square, computing the initial vector $\sigma^{0}=\left[\sigma_{0}^{0}, \sigma_{1}^{0}, \sigma_{2}^{0}, \ldots, \sigma_{N}^{0}\right]^{T}$ from the initial condition $u(x, 0)=g_{0}(x)$ given, $(N+1)$ equation in $(N+3)$ unknowns, to determine these unknown function, the following relations at the knots are used

$$
\begin{aligned}
& U_{x}(0,0)=u_{x}\left(x_{0}, 0\right) \\
& U\left(x_{i}, 0\right)=g_{0}\left(x_{i}\right), \quad i=1(1)(N-1) \\
& U_{x}(L, 0)=u_{x}\left(x_{N}, 0\right) .
\end{aligned}
$$

We have the tridiagonal system of equation that can be solved by: $R \sigma^{0}=E$, where

$$
R=\left[\begin{array}{cccccccccc}
4 & 2 & 0 & & & & & & & \\
1 & 4 & 1 & & & & & & & \\
& 1 & 4 & 1 & & & & & & \\
& & & & \ddots & \ddots & \ddots & & & \\
& & & & & & & & & \\
& & & & & & & 0 & 4 & 1 \\
& & & & & & & 0 & 4
\end{array}\right],
$$

which can be solved by using Thomas algorithm [12]. 


\section{The Stability}

To learn the stability of the proposed method, we will rewrite the equation (21) in terms of the nodal parameters $\sigma_{m}^{n}$ and $f(\eta, t)=0$ [17] so get

$$
\begin{aligned}
& \ell_{1} \sigma_{m-2}^{n+1}+\ell_{2} \sigma_{m-1}^{n+1}+\ell_{3} \sigma_{m}^{n+1}+\ell_{3} \sigma_{m+1}^{n+1}+\ell_{2} \sigma_{m+2}^{n+1}+\ell_{1} \sigma_{m+3}^{n+1}=\ell_{4} \sigma_{m-2}^{n}+ \\
& \ell_{5} \sigma_{m-2}^{n}+\ell_{6} \sigma_{m}^{n}+\ell_{7} \sigma_{m+1}^{n}+\ell_{8} \sigma_{m+2}^{n}+\ell_{9} \sigma_{m+3}^{n},
\end{aligned}
$$

where

$$
\begin{aligned}
& \ell_{1}=\left(\frac{79}{4200}\right)-\left(\frac{\lambda^{2}}{6}+\frac{\beta}{20}\right)+2 \beta^{2}, \quad \ell_{2}=\left(\frac{1501}{1400}\right)-\left(\frac{3 \lambda^{2}}{2}+\frac{9 \beta}{20}\right)-6 \beta^{2}, \\
& \ell_{3}=\left(\frac{11929}{2100}\right)+\left(\frac{5 \lambda^{2}}{3}+\frac{\beta}{2}\right)+4 \beta^{2}, \quad \ell_{4}=\left(\frac{53}{8400}\right)-\left(-\frac{\lambda^{2}}{12}+\frac{3 \beta}{8}\right)-\beta^{2}-\left(\frac{17 \lambda}{200}\right), \\
& \ell_{5}=\left(\frac{1007}{2800}\right)-\left(-\frac{3 \lambda^{2}}{4}+\frac{27 \beta}{8}\right)+3 \beta^{2}-\left(\frac{17 \lambda}{8}\right), \\
& \ell_{6}=\left(\frac{8003}{4200}\right)+\left(-\frac{5 \lambda^{2}}{6}+\frac{15 \beta}{4}\right)-2 \beta^{2}-\left(\frac{17 \lambda}{5}\right), \\
& \ell_{7}=\left(\frac{8003}{4200}\right)+\left(-\frac{5 \lambda^{2}}{6}+\frac{15 \beta}{4}\right)-2 \beta^{2}+\left(\frac{17 \lambda}{5}\right), \\
& \ell_{8}=\left(\frac{1007}{2800}\right)-\left(-\frac{3 \lambda^{2}}{4}+\frac{27 \beta}{8}\right)+3 \beta^{2}+\frac{17 \lambda}{8}, \\
& \ell_{9}=\left(\frac{53}{8400}\right)-\left(-\frac{\lambda^{2}}{12}+\frac{3 \beta}{8}\right)-\beta^{2}+\left(\frac{17 \lambda}{200}\right) .
\end{aligned}
$$

By applying the Von-Neumann method [10], of equation (22) for any, $0 \leq \eta \leq 1$ and

$$
\sigma_{m}^{n}=\ddot{\Upsilon}^{n} e^{i \beta m h},
$$

where $\ddot{Y}$ represents the time dependence of the solution, and the exponential function shows that the spatial dependence such that $\beta h$ represents the position along the grid and $i$ is $\sqrt{-1}$. By substituting, $\sigma_{m}^{n}$, into (22), we get:

$$
\begin{aligned}
& \ell_{1} \ddot{\Upsilon}^{n+1} e^{i \beta(m-2) h}+\ell_{2} \ddot{\Upsilon}^{n+1} e^{i \beta(m-1) h}+\ell_{3} \ddot{\Upsilon}^{n+1} e^{i \beta m h}+\ell_{3} \ddot{\Upsilon}^{n+1} e^{i \beta(m+1) h}+ \\
& \ell_{2} \ddot{\Upsilon}^{n+1} e^{i \beta(m+2) h}+\ell_{1} \ddot{\Upsilon}^{n+1} e^{i \beta(m+3) h}=\ell_{4} \ddot{\Upsilon}^{n} e^{i \beta(m-2) h}+\ell_{5} \ddot{\Upsilon}^{n} e^{i \beta(m-1) h}+ \\
& \ell_{6} \ddot{\Upsilon}^{n} e^{i \beta m h}+\ell_{7} \ddot{\Upsilon}^{n} e^{i \beta(m+1) h}+\ell_{8} \ddot{\Upsilon}^{n} e^{i \beta(m+2) h}+\ell_{9} \ddot{\Upsilon}^{n} e^{i \beta(m+3) h} .
\end{aligned}
$$

By dividing equation (23) by $\ddot{\Upsilon}^{n} e^{i \beta m h}$, we have

$$
\ddot{\Upsilon}=\frac{\left(\ell_{4} e^{-2 i \beta h}+\ell_{5} e^{-i \beta h}+\ell_{6}+q_{7} e^{i \beta h}+\ell_{8} e^{2 i \beta h}+\ell_{9} e^{3 i \beta h}\right)}{\left(\ell_{1} e^{-2 i \beta h}+\ell_{2} e^{-i \beta h}+\ell_{3}+\ell_{3} e^{i \beta h}+\ell_{2} e^{2 i \beta h}+\ell_{1} e^{3 i \beta h}\right)^{.}} .
$$


After some simplifications, get $|\ddot{Y}|<1$, so cubic B-spline least square method with quadratic weight function for PIDE is unconditionally stable.

\section{Numerical Examples}

In this section, we will apply the scheme described in Section 3 to test two examples to demonstrate the efficiency, accuracy, and applicability of the present scheme. Results obtained by this scheme are compared with the analytical solution of each example by computing the maximum norm error $L_{\infty}$ and norm error $L_{2}$.

Let, $t_{n}=n k, n=0(1) M$, where $M$ denoted the final time level $t_{M}$ and $N+1$ is the number of the nodes to check the accuracy of the proposed method, where

$$
\begin{aligned}
& L_{\infty}=\max _{0 \leq i \leq N}\left|u\left(x_{i}, t_{M}\right)-U_{i}^{M}\right|, \\
& L_{2}=\frac{1}{N}\left(\sum_{i=0}^{N}\left|u\left(x_{i}, t_{M}\right)-U_{i}^{M}\right|^{2}\right)^{\frac{1}{2}} .
\end{aligned}
$$

Example 1. [15]

$$
\begin{gathered}
u_{t}(x, t)+m u_{x}(x, t)-b u_{x x}(x, t)=\int_{0}^{t}(t-s)^{-\propto} u(x, s) d s+f(x, t) \\
x \in[0,1], \quad \propto=\frac{1}{4}, \quad t>0, \quad m=0.5, \quad b=0.001 .
\end{gathered}
$$

The initial and boundary conditions are

$$
\begin{array}{ll}
u(x, 0)=2 \sin ^{2} \pi x, & 0 \leq x \leq 1 \\
u(0, t)=u(1, t)=0, & 0 \leq t \leq T .
\end{array}
$$

The exact solution is:

$$
u(x, t)=2\left(t^{2}+t+1\right) \sin ^{2} \pi x .
$$

Example 2. [15]

$$
\begin{gathered}
u_{t}(x, t)+m u_{x}(x, t)-b u_{x x}(x, t)=\int_{0}^{t}(t-s)^{-\propto} u(x, s) d s+f(x, t) \\
x \in[0,1], \quad \propto=\frac{1}{3}, \quad t>0, \quad m=000.5, \quad b=0.5 .
\end{gathered}
$$

The initial and boundary conditions are

$$
\begin{aligned}
& u(x, 0)=1-\cos 2 \pi x+2 \pi^{2} x(1-x), \quad 0 \leq x \leq 1 \\
& u(0, t)=(t+1), \quad u(1, t)=-(t+1), \quad t \geq 0 .
\end{aligned}
$$


The exact solution is:

$$
u(x, t)=(t+1)^{2}\left(1-\cos 2 \pi x+2 \pi^{2} x(1-x)\right) .
$$

Table 1. $L_{\infty}$ and $L_{2}$ at $\Delta t=0.00001$ of Example 1 .

\begin{tabular}{|c|c|c|c|}
\hline$h$ & $M$ & $L_{2},(\Delta t=0.00001)$ & $L_{\infty},(\Delta t=0.00001)$ \\
\hline \multirow[t]{3}{*}{0.01} & 10 & $2.4908 \mathrm{e}-10$ & $6.8233 \mathrm{e}-09$ \\
\hline & 50 & $2.8120 \mathrm{e}-10$ & $6.8264 \mathrm{e}-09$ \\
\hline & 100 & $3.2734 \mathrm{e}-10$ & $6.8304 \mathrm{e}-09$ \\
\hline \multirow[t]{3}{*}{0.0066} & 10 & $1.8637 \mathrm{e}-11$ & $5.9803 \mathrm{e}-10$ \\
\hline & 50 & $2.7995 \mathrm{e}-11$ & $5.9844 \mathrm{e}-10$ \\
\hline & 100 & $4.1542 \mathrm{e}-11$ & $7.4737 \mathrm{e}-10$ \\
\hline \multirow[t]{3}{*}{0.005} & 10 & $3.2603 e-12$ & $1.0624 \mathrm{e}-10$ \\
\hline & 50 & $7.1431 \mathrm{e}-12$ & $1.5080 \mathrm{e}-10$ \\
\hline & 100 & $1.2427 \mathrm{e}-11$ & $2.7452 \mathrm{e}-10$ \\
\hline \multirow[t]{3}{*}{0.004} & 10 & $9.4854 \mathrm{e}-13$ & $2.7797 \mathrm{e}-11$ \\
\hline & 50 & $2.8404 \mathrm{e}-12$ & 7.0082e-11 \\
\hline & 100 & $5.3032 \mathrm{e}-12$ & $1.3399 \mathrm{e}-10$ \\
\hline \multirow[t]{3}{*}{0.0033} & 10 & $3.8475 \mathrm{e}-13$ & $9.5847 \mathrm{e}-12$ \\
\hline & 50 & $1.4113 \mathrm{e}-12$ & $3.8918 \mathrm{e}-11$ \\
\hline & 100 & $2.7190 \mathrm{e}-12$ & $7.5974 \mathrm{e}-11$ \\
\hline
\end{tabular}




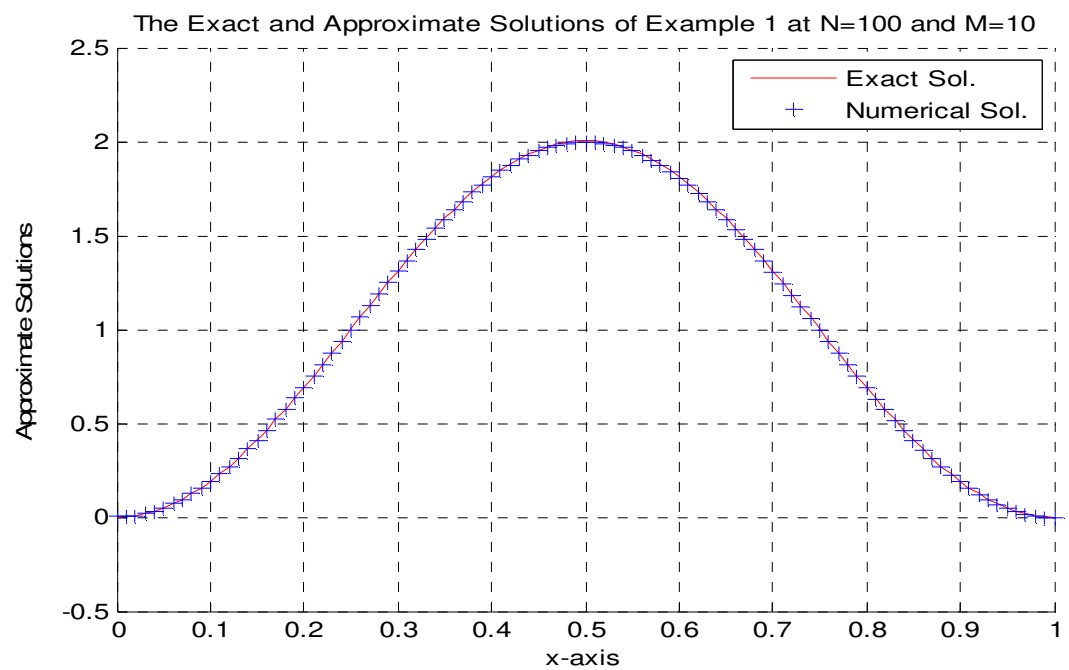

Table 2. $L_{\infty}$ and $L_{2}$ at $\Delta t=0.00001$ of Example 2 .

\begin{tabular}{|c|c|c|c|}
\hline$h$ & $M$ & $L_{2},(\Delta t=0.00001)$ & $L_{\infty},(\Delta t=0.00001)$ \\
\hline \multirow{3}{*}{0.01} & 10 & $2.2113 \mathrm{e}-09$ & $5.6587 \mathrm{e}-08$ \\
\cline { 2 - 4 } & 50 & $2.5080 \mathrm{e}-09$ & $5.6903 \mathrm{e}-08$ \\
\cline { 2 - 4 } & 100 & $2.9080 \mathrm{e}-09$ & $5.7298 \mathrm{e}-08$ \\
\hline \multirow{5}{*}{0.0066} & 10 & $1.6652 \mathrm{e}-10$ & $5.1153 \mathrm{e}-09$ \\
\cline { 2 - 4 } & 50 & $2.4548 \mathrm{e}-10$ & $5.1777 \mathrm{e}-09$ \\
\cline { 2 - 4 } & 100 & $3.5394 \mathrm{e}-10$ & $5.7728 \mathrm{e}-09$ \\
\cline { 2 - 4 } & 10 & $2.9026 \mathrm{e}-11$ & $9.1667 \mathrm{e}-10$ \\
\cline { 2 - 4 } & 100 & $6.0316 \mathrm{e}-11$ & $1.1463 \mathrm{e}-09$ \\
\hline \multirow{5}{*}{0.004} & 10 & $1.0189 \mathrm{e}-10$ & $1.9974 \mathrm{e}-09$ \\
\cline { 2 - 4 } & 50 & $8.3402 \mathrm{e}-12$ & $2.4111 \mathrm{e}-10$ \\
\cline { 2 - 4 } & 100 & $2.3318 \mathrm{e}-11$ & $5.1065 \mathrm{e}-10$ \\
\hline \multirow{3}{*}{0.0033} & 10 & $4.2636 \mathrm{e}-11$ & $9.5255 \mathrm{e}-10$ \\
\cline { 2 - 4 } & 50 & $3.3180 \mathrm{e}-12$ & $8.0990 \mathrm{e}-11$ \\
\cline { 2 - 4 } & 100 & $1.1394 \mathrm{e}-11$ & $2.7797 \mathrm{e}-10$ \\
\hline
\end{tabular}




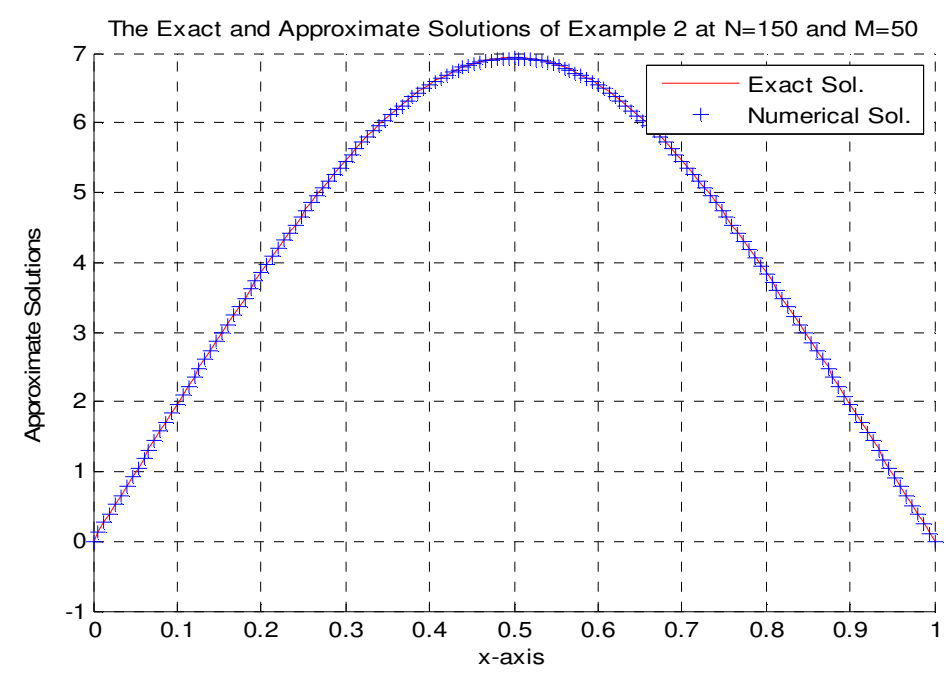

\section{Conclusions}

In this paper, we introduced a new numerical scheme to solving the integrodifferential equations with the weakly singular kernel by using the cubic B-spline leastsquare method with quadratic B-spline as a weight function. The method was performed when taking values $N=100,150,200,250$ and 300 with $\Delta t=0.00001$ with a different $M$, which presented in Tables 1-2. From Figures 1-2, the numerical and the exact solutions are very harmonic which signalizes the numerical solutions effectively. We calculated $L_{2}$ and $L_{\infty}$ norms errors varied to test the accuracy of the proposed method, also, the numerical results are in good agreement with the exact solutions. The proposed method is an effective and unconditionally stable method.

\section{References}

[1] A. Chakrabarti and S. C. Martha, Approximate solutions of Fredholm integral equations of the second kind, Appl. Math. Comput. 211 (2009), 459-466. https://doi.org/10.1016/j.amc.2009.01.088

[2] I. Dağ, Least-squares quadratic B-spline finite element method for the regularized long wave equation, Comput. Methods Appl. Mech. Engrg. 182 (2000), 205-215. https://doi.org/10.1016/S0045-7825(99)00106-1

[3] I. Dağ and M. Naci Özer, Approximation of the RLW equation by the least square cubic B-spline finite element method, Applied Mathematical Modelling 25 (2001), 221-231. https://doi.org/10.1016/S0307-904X(00)00030-5 
[4] M. Dehghan, Weighted finite difference techniques for the one-dimensional advectiondiffusion equation, Appl. Math. Comput. 147 (2004), 307-319. https://doi.org/10.1016/S0096-3003(02)00667-7

[5] S. Dhawan, S. Kumar, and S. Kapoor, Approximation of Burgers equation using B-spline finite element method, Int. J. Appl. Math. Mech. 7(17) (2011), 61-86.

[6] S. Dhawan, S. Kumar Bhowmik and S. Kumar, Galerkin-least square B-spline approach toward advection-diffusion equation, Appl. Math. Comput. 261 (2015), 128-140. https://doi.org/10.1016/j.amc.2015.03.092

[7] L. R. T. Gardner, G. A. Gardner and A. Dogan, A least-squares finite element scheme for the RLW equation, Comm. Numer. Methods Engrg. 12 (1996), 795-804. https://doi.org/10.1002/(SICI)1099-0887(199611)12:11<795::AID-CNM22>3.0.CO;2-O

[8] R. El Jid, Moving least squares and Gauss Legendre for solving the integral equations of the second kind, IAENG Int. J. Appl. Math. 49(1) (2019), 90-97.

[9] S. Kutluay, A. Esen and I. Dag, Numerical solutions of the Burgers' equation by the least-squares quadratic B-spline finite element method, J. Comput. Appl. Math. 167 (2004), 21-33. https://doi.org/10.1016/j.cam.2003.09.043

[10] A. R. Mitchell and D. F. Griffiths, The Finite Difference Method in Partial Differential Equations, John Wiley \& Sons, New York, 1980.

[11] H. Nguyen and J. Reynen, A space-time least-square finite element scheme for advection- diffusion equation, Computer Methods in Applied Mechanics and Engineering 42 (1984), 331-342. https://doi.org/10.1016/0045-7825(84)90012-4

[12] J. Noye, Numerical Solutions of Partial Differential Equation, North Holland Publishing Company, Amsterdam, New York, Oxford, 1982.

[13] P. M. Prenter, Spline and Variational Method, John Wiley and Sons, New York, NY, USA, 1975.

[14] S. N. Shehab, H. Adel Ali and H. Mohammed Yaseen, Least squares method for solving integral equations with multiple time lags, Eng. and Tech. Journal 28(10) (2010), 18931899.

[15] S. S. Siddiqi and S. Arshad, Numerical solution of convection-diffusion integrodifferential equations with a weakly singular kernel, Journal of Basic and Applied Scientific Research 3(11) (2013), 106-120.

[16] D. B. Spalding, A novel finite difference formulation for differential expressions involving both first and second derivatives, International Journal for Numerical Method in Engineering 4 (1972), 551-559. https://doi.org/10.1002/nme.1620040409 
[17] W. Tuzikiewics and M. Duda, Bioheat transfer equation. The problem of FDM explicit scheme stability, Journal of Applied Mathematics and Computation Mechanics 14(4) (2015), 139-144. https://doi.org/10.17512/jamcm.2015.4.14

[18] Q. Wang, Keyan Wang and Shaojun Chen, Least squares approximation method for the solution of Volterra-Fredholm integral equations, J. Comput. Appl. Math. 272 (2014), 141-147. https://doi.org/10.1016/j.cam.2014.05.010 